\title{
PARTIAL AUTOMORPHISMS AND THE RECONSTRUCTION CONJECTURE
}

\author{
W. L. KOCAY \\ (Received 20 February 1982) \\ Communicated by W. D. Wallis
}

\begin{abstract}
Let $\Gamma$ be a graph with isomorphic subgraphs $G$ and $H$, and let $\theta: G \rightarrow H$ be an isomorphism. If $\theta$ can be extended to an automorphism of $\Gamma$, we call $\theta$ a partial automorphism of $\Gamma$.

We consider the application of partial automorphisms to the graph reconstruction conjecture, in particular, to the problem of reconstructing graphs with two vertices of degree $k-1$ and the remaining vertices of degree $k$.
\end{abstract}

1980 Mathematics subject classification (Amer. Math. Soc.): 05 C 60.

\section{Introduction}

We study the application of partial automorphisms to the reconstruction of "almost" regular graphs, that is, graphs with $n$ vertices of degree $k$ and two vertices of degree $k-1$, where $k \geqslant 3$, so that the total number of vertices is $n+2$.

1.1 Definition. Let $G$ and $H$ have the same number of vertices. $G$ and $H$ are reconstructions of each other if there exists a bijection $\theta: V(G) \rightarrow V(H)$ such that the vertex-deleted subgraph $G-v \simeq H-\theta(v)$, for every $v \in V(G)$.

1.2 Definition. $G$ is reconstructible if $G \simeq H$ whenever $G$ and $H$ are reconstructions of each other.

We consider only connected graphs, since it is well-known that disconnected graphs are reconstructible [1].

(9) 1984 Australian Mathematical Society $0263-6115 / 84 \$ A 2.00+0.00$ 
It is a simple observation that all $k$-regular graphs are reconstructible, and that if $G$ has only two degrees, we can take these to be $k$ and $k-1$; otherwise $G$ is reconstructible. Again, if $G$ has only one vertex of degree $k-1$, then $G$ is reconstructible.

Accordingly, let $G$ have $n$ vertices of degree $k$ and two vertices, $u$ and $v$, of degree $k-1$, where $k \geqslant 3$, since $k=2$ is not very interesting. The special case $k=3$ is treated in [3] and a closely related problem is considered in [2]. If $u v \in E(G)$, then $G-u$ has one vertex of degree $k-2$ (that is, $v$ ) and $k-2$ vertices of degree $k-1$. There is only one way to rejoin $u$ to get the proper degree sequence, so that $G$ is reconstructible.

If $u v \notin E(G)$, then there are $k$ ways to rejoin $u$ to $G-u$ to get the correct degree sequence. So let $H$ be obtained from $G-u$ by adjoining a new vertex $x$ adjacent to $v$ and to any $k-2$ of the other $k-1$ vertices of degree $k-1$ in $G-u$. Then $\operatorname{deg}_{H} x=k-1$. Call the other vertex of degree $k-1$ in $H w$. This is illustrated below. Vertices of degree $k-1$ are marked by dark dots.

If $G$ is not reconstructible, then without loss of generality, we can take $H$ as a reconstruction of $G$.
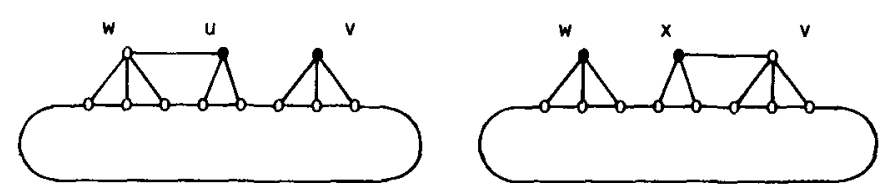

Figure 1.1

We have $G-u=H-x$, and since $G$ and $H$ are reconstructions of each other, we must have $G-v \simeq H-w$, since $v$ and $w$ are the other vertices of degree $k-1$ in $G$ and $H$, respectively (see [1]). Accordingly, let $p: G-v \rightarrow H-w$ be an isomorphism.

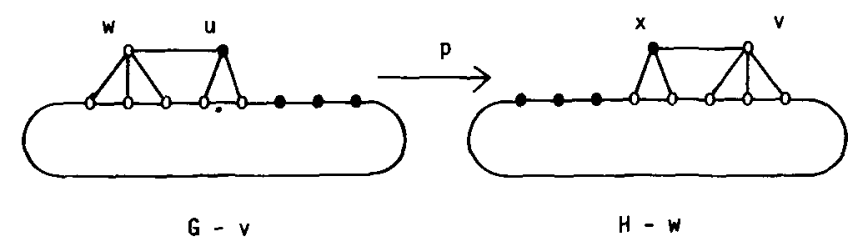

Figure 1.2

1.3 LEMMA. There exist positive integers $k_{1}$ and $k_{2}$ such that either:

(i) $p^{k_{1}}(u)=x$ and $p^{k_{2}}(w)=v$; or

(ii) $p^{k_{1}}(u)=v$ and $p^{k_{2}}(w)=x$.

Proof. Let $V=V(G) \cup V(H)$. Then $p: V-\{v, x\} \rightarrow V-\{u, w\}$. Consider $p(u)$. Either $p(u) \in\{v, x\}$, or we can find $p^{2}(u)$. Either $p^{2}(u) \in\{v, x\}$, or we 
can find $p^{3}(u)$. Since $p$ is one-to-one and onto, and $V$ is finite, we must eventually have $p^{k_{1}}(u) \in\{v, x\}$. Similarly, $p^{k_{2}}(w) \in\{v, x\}$.

1.4 Definition. Let $\Gamma$ be a graph with isomorphic subgraphs $G$ and $H$ and let $\theta: G \rightarrow H$ be an isomorphism. If $\theta$ can be extended to an automorphism of $\Gamma$ then $\theta$ is a partial automorphism of $\Gamma$.

We will embed $G$ and $H$ into a graph $\Gamma$ for which $p$ of Lemma 1.3 becomes a partial automorphism of $\Gamma$. This will enable us to determine much of the structure of $G$ and $H$.

For let $\Gamma=G+u v$. Then clearly, $H+w x \simeq \Gamma$, by Figures 1.1 and 1.3. Let $\psi$ be the natural isomorphism between $H+w x$ and $\Gamma$, that is, $\psi(x)=u$ and $\psi(y)=y$ for $y \neq x$. Since $\Gamma-v=G-v \simeq H-w \simeq \Gamma-w$, we have $\theta=\psi p$ : $\Gamma-v \rightarrow \Gamma-w$ is an isomorphism, where the product $\psi p$ is read from right to left.

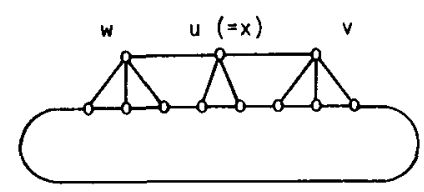

Figure 1.3

1.5 LeMma. $\theta$ is a partial automorphism of $\Gamma$.

Proof. $\theta$ maps $V(\Gamma)-\{v\}$ to $V(\Gamma)-\{w\}$. Extend $\theta$ to $V(\Gamma)$ by putting $\theta(v)=w$. To show that $\theta$ is an automorphism of $\Gamma$, notice that $\theta$ maps $\Gamma-v$ to $\Gamma-w$. Therefore $\theta$ maps the vertices of degree $k-1$ in $\Gamma-v$ to those of degree $k-1$ in $\Gamma-w$, that is, it maps the vertices adjacent to $v$ in $\Gamma$ to those adjacent to $w$ in $\Gamma$. Therefore the extension $\theta(v)=w$ completes $\theta$ to an automorphism of $\Gamma$.

This automorphism of $\Gamma$ will enable us to determine much of the structure of $G$ and $H$. In some cases it determines $G$ and $H$ completely.

$$
\text { 2. } p^{k_{1}}(u)=x \text { and } p^{k_{2}}(w)=v
$$

We assume throughout this section that the first alternative of Lemma 1.3 holds, namely that $p^{k_{1}}(u)=x$ and $p^{k_{2}}(w)=v$. In this case the vertices $u$ and $w$ lie in different orbits of $\langle\theta\rangle$. 
Let $U$ be the orbit of $u$ and $W$ the orbit of $w$, that is, $U=$ $\left\{u, \theta(u), \theta^{2}(u), \ldots, \theta^{k_{1}-1}(u)\right\}$ and $W=\left\{w, \theta(w), \theta^{2}(w), \ldots, \theta^{k_{2}}(w)=v\right\}$. If we write $u_{i}=\theta^{i}(u)$, for $i=0,1,2, \ldots, k_{1}-1$, and $w_{i}=\theta^{i}(w)$, for $i=0,1,2, \ldots, k_{2}$, then $U=\left\{u_{0}, u_{1}, \ldots, u_{k_{1}-1}\right\}$ and $W=\left\{w_{0}, w_{1}, \ldots, w_{k_{2}}\right\}$, where $w_{k_{2}}=v \cdot|U|=k_{1}$ and $|W|=k_{2}+1$.

Let $g=\operatorname{gcd}\left(k_{1}, k_{2}+1\right)$.

2.1 Definition. We define a bipartite graph $G^{\prime}\left(k_{1}, k_{2}\right)$ with vertices $A=$ $\left\{a_{0}, a_{1}, \ldots, a_{k_{1}-1}\right\}$ and $B=\left\{b_{0}, b_{1}, \ldots, b_{k_{2}}\right\}$. Vertex $a_{i}$ is joined to $b_{i}, b_{i+g}$, $b_{i+2 g}, \ldots$, and to $b_{i-1}, b_{i+g-1}, b_{i+2 g-1}, \ldots$, for $i=0, \ldots, k_{1}-1$, and where the arithmetic is computed modulo $k_{2}+1$.

Alternatively, we could have defined $G^{\prime}\left(k_{1}, k_{2}\right)$ by saying that vertex $b_{i}$ is joined to $a_{i}, a_{i+g}, a_{i+2 g}, \ldots$, and to $a_{i+1}, a_{i+g+1}, a_{i+2 g+1}, \ldots$, for $i=0,1, \ldots, k_{2}$, and the arithmetic is computed modulo $k_{1}$.

2.2 LeMMA. The induced subgraph $\Gamma[U \cup W]$ contains a spanning subgraph isomorphic to $G^{\prime}\left(k_{1}, k_{2}\right)$.

Proof. The mapping $a_{i} \rightarrow u_{i}, i=0,1, \ldots, k_{1}-1$ and $b_{i} \rightarrow w_{i}, i=0,1, \ldots, k_{2}$, is an embedding of $G^{\prime}\left(k_{1}, k_{2}\right)$ into $\Gamma[U \cup W]$. For $u\left(=u_{0}\right)$ is joined to $w\left(=w_{0}\right)$ and to $v\left(=w_{k_{2}}\right)$. Since $|U|=k_{1},|W|=k_{2}+1, g=\operatorname{gcd}\left(k_{1}, k_{2}+1\right)$, and $\theta$ is an automorphism of $\Gamma[U \cup W]$, we see that the above embedding is indeed an isomorphism.

By Lemma 2.2 we can consider $G^{\prime}\left(k_{1}, k_{2}\right)$ as a subgraph of $\Gamma[U \cup W]$. We will identify the sets $A$ with $U$ and $B$ with $W$, and consider $\theta$ as acting on $G^{\prime}\left(k_{1}, k_{2}\right)$ as an automorphism.

If $g>1$, there are two orbits of edges: the orbit containing $u_{0} w_{0}, u_{0} w_{g}$, $u_{0} w_{2 g}, \ldots$, and its translations under $\theta$; and the orbit containing $u_{0} w_{k_{2}}$, $u_{0} w_{2 g-1}, \ldots$, and its translations under $\theta$. The first orbit contains the edge $u w$ $\left(=u_{0} w_{0}\right)$ and the second orbit contains $u v\left(=u_{0} w_{k_{2}}\right)$.

2.3 THEOREM. If $g=\operatorname{gcd}\left(k_{1}, k_{2}+1\right)=1$, then $G \simeq H$.

PROof. If $g=1$ there is only one orbit of edges, since $u_{0}$ is joined to all vertices of $W$. Therefore $G=\Gamma-u v \simeq \Gamma-u w \simeq H$.

Thus, in particular, if $k_{1}=k_{2}$, then $g=1$, and $G \simeq H$. 
If $g>1$, then the degree of $u_{i}$ in $G^{\prime}\left(k_{1}, k_{2}\right)$ is $2\left(k_{2}+1\right) / g$ and the degree of $w_{i}$ is $2 k_{1} / g . G^{\prime}\left(k_{1}, k_{2}\right)$ is illustrated in Figure 2.1 where the two edge-orbits are shown in solid and broken lines.

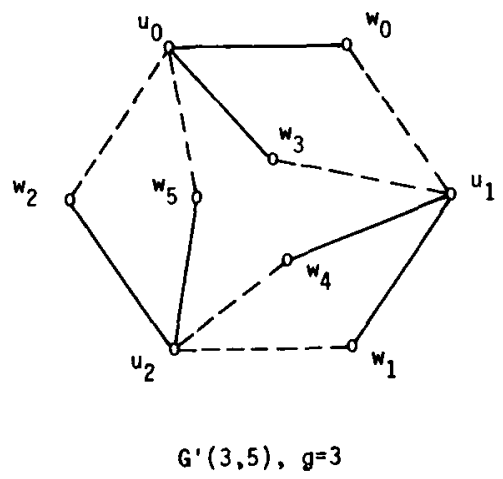

Figure 2.1

$G^{\prime}\left(k_{1}, k_{2}\right)$ is a regular graph only when $2 k_{1} / g=2\left(k_{2}+1\right) / g$, that is, when $k_{1}=k_{2}+1$. In this case $g=k_{1}=k_{2}+1$, and $G^{\prime}\left(k_{1}, k_{1}-1\right)$ is a 2-regular graph. In fact it is a polygon of length $2 k_{1}$ in which alternate vertices and edges belong to different orbits of $\langle\theta\rangle$. Since $\Gamma$ is $k$-regular, where $k \geqslant 3$, we gain very little information about $G$ and $H$.

When $k=3$, we must have $g=k_{1}=k_{2}+1$, for otherwise the degree of $u_{i}$ or $w_{i}$ would be at least 4 .

$$
\text { 3. } p^{k_{1}}(u)=v \text { and } p^{k_{2}}(w)=x
$$

We assume throughout this section that the second alternative of Lemma 1.3 holds, namely $p^{k_{1}}(u)=v$ and $p^{k_{2}}(w)=x$.

\subsection{THEOREM. If $k_{1}=k_{2}$, then $G \simeq H$.}

Proof. Consider the orbit of $\langle\theta\rangle$ containing $u$ in $\Gamma$. It contains $u$, $\theta(u), \theta^{2}(u), \ldots, \theta^{k_{1}}(u)=v, \theta(v)=w, \theta(w), \theta^{2}(w), \ldots, \theta^{k_{2}}(w)=u$. The orbit contains $k_{1}+k_{2}+1=2 k_{1}+1$ vertices. Consider the edge $u v \in E(\Gamma)$. $\theta^{k_{1}+1}(u v)=\theta^{k_{1}+1}(u) \theta^{k_{1}+1}(v)=w u$. Therefore, $G=\Gamma-u v \simeq \Gamma-u w \simeq H$, since edges $u v$ and $u w$ are in the same edge-orbit of $\langle\theta\rangle$.

3.2 Definition. We define a graph $G\left(k_{1}, k_{2}\right)$ with vertices $0,1,2, \ldots, k_{1}+k_{2}$ such that vertex $i$ is adjacent to vertices $i+k_{1}, i-k_{1}, i+k_{1}+1$, and $i-k_{1}-1$, where the arithmetic is calculated modulo $k_{1}+k_{2}+1$. 
Properties of the graph $G\left(k_{1}, k_{2}\right)$ are important for the following reason. Let $U$ denote the orbit of $\langle\theta\rangle$ containing $u$. Then $U=\left\{u, \theta(u), \theta^{2}(u), \ldots, \theta^{k_{1}}(u)=v\right.$, $\left.\theta(v)=w, \theta(w), \ldots, \theta^{k_{2}}(w)=u\right\}$. Write $u_{i}=\theta^{i}(u)$, for $i=0,1,2, \ldots, k_{1}+k_{2}$. Then $u=u_{0}, v=u_{k_{1}}$, and $w=u_{k_{1}+1}$, and $U=\left\{u_{0}, u_{1}, \ldots, u_{k_{1}+k_{2}}\right\}$.

3.3 LEMMA. The induced subgraph $\Gamma[U]$ contains a spanning subgraph isomorphic to $G\left(k_{1}, k_{2}\right)$.

Proof. The correspondence $\sigma: G\left(k_{1}, k_{2}\right) \rightarrow \Gamma[U]$ given by $\sigma(i)=u_{i}, i=$ $0,1, \ldots, k_{1}+k_{2}$ embeds an isomorphic copy of $G\left(k_{1}, k_{2}\right)$ into $\Gamma[U]$; for $u\left(=u_{0}\right)$ is adjacent to $v\left(=u_{k_{1}}\right)$ and to $w\left(=u_{k_{1}+1}\right)$, and $\theta$ is an automorphism of $\Gamma[U]$.

In view of Lemma 3.3, we will consider $G\left(k_{1}, k_{2}\right)$ as a subgraph of $\Gamma[U]$, with vertices $u_{0}, u_{1}, \ldots, u_{k_{1}+k_{2}}$ on which $\theta$ acts according to the rule $\theta\left(u_{i}\right)=u_{i+1}$, where the subscript is computed modulo $k_{1}+k_{2}+1$.

Notice that $G\left(k_{1}, k_{2}\right)$ consists of an edge-disjoint union of polygons. For the sequence $u_{0}, u_{k_{1}}, u_{2 k_{1}}, u_{3 k_{1}}, \ldots, u_{0}$ gives a polygon of length $\left(k_{1}+k_{2}+1\right) / g_{1}$, where $g_{1}=\operatorname{gcd}\left(k_{1}, k_{1}+k_{2}+1\right)=\operatorname{gcd}\left(k_{1}, k_{2}+1\right)$. The translations of this polygon under $\theta$ give a set of $g_{1}$ vertex-disjoint polygons.

Similarly, the sequence $u_{0}, u_{k_{1}+1}, u_{2\left(k_{1}+1\right)}, \ldots, u_{0}$ gives a polygon of length $\left(k_{1}+k_{2}+1\right) / g_{2}$, where $g_{2}=\operatorname{gcd}\left(k_{1}+1, k_{1}+k_{2}+1\right)=\operatorname{gcd}\left(k_{1}+1, k_{2}\right)$. Its translations under $\theta$ give a set of $g_{2}$ vertex-disjoint polygons, which are edge-disjoint from the first set.

These two sets of polygons decompose the edge set of $G\left(k_{1}, k_{2}\right)$ into two edge-orbits of $\langle\theta\rangle$. The first set is the orbit containing the edge $u_{0} u_{k_{1}}=u v$ and the second set contains $u_{0} u_{k_{1}+1}=u w$. Since $G=\Gamma-u v$ and $H \simeq \Gamma-u w$, we cannot use the argument of Theorem 2.1 to prove that $G \simeq H$.

By Theorem 2.1, we need not consider the case $k_{1}=k_{2}$ anymore.

3.4 THEOREM. If $\left|k_{1}-k_{2}\right|=1$, then $G\left(k_{1}, k_{2}\right)$ is 3-regular. If $\left|k_{1}-k_{2}\right| \geqslant 2$, then $G\left(k_{1}, k_{2}\right)$ is 4-regular.

Proof. Suppose that $k_{1}=k_{2}+1$. Then $k_{1}+k_{2}+1=2 k_{1}$ and $g_{1}=k_{1}$. The orbit of $u v$ consists of $g_{1}$ "polygons" of length 2 . In this case $g_{2}=$ $\operatorname{gcd}\left(k_{1}+1, k_{2}\right)=\operatorname{gcd}\left(k_{2}+2, k_{2}\right)$. If $k_{2}$ is odd, then $g_{2}=1$, and the orbit of $u w$ is a single polygon of length $2 k_{1}$. In this case $G\left(k_{1}, k_{2}\right)$ is a polygon of length $2 k_{1}$ with main diagonals present.

If $k_{2}$ is even, then $g_{2}=2$, and the orbit of $u w$ breaks into two polygons, each of length $k_{1} \cdot G\left(k_{1}, k_{2}\right)$ is then a $k_{1}$-prism, that is, two polygons with corresponding vertices joined by a matching. 
Now let $k_{1}=k_{2}-1$. Then $k_{1}+k_{2}+1=2 k_{2}$. This case is equivalent to the above. Thus, if $\left|k_{1}-k_{2}\right|=1, G\left(k_{1}, k_{2}\right)$ is one of the 3-regular graphs described above. This is illustrated in Figure 3.1. The two orbits of edges are shown in solid and broken lines.

Finally, if $\left|k_{1}-k_{2}\right| \geqslant 2$, then degenerate "polygons" of length 2 do not occur, and $G\left(k_{1}, k_{2}\right)$ is a 4-regular graph.
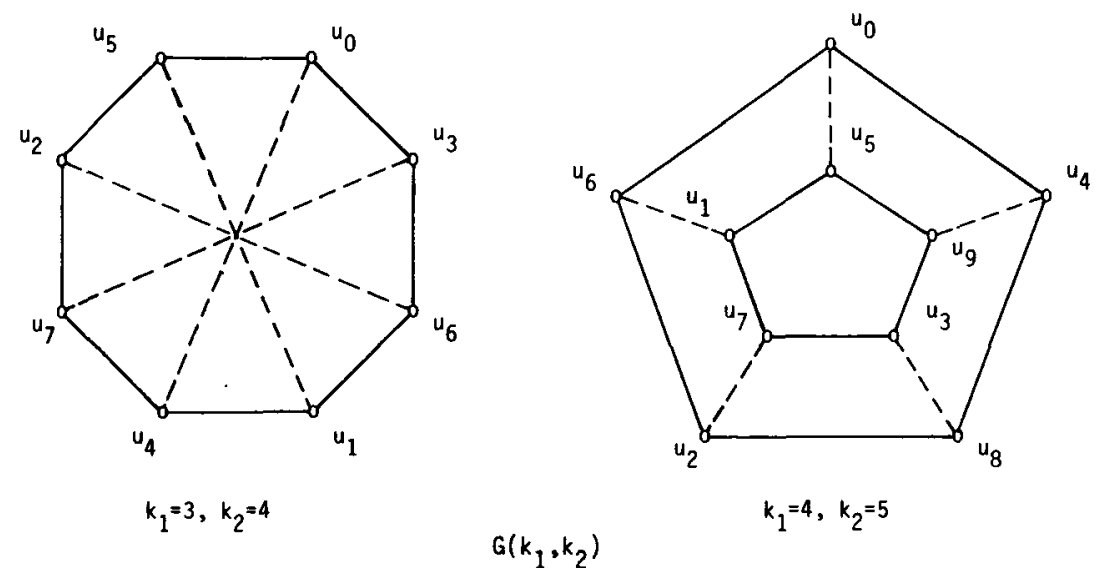

Figure 3.1

3.5 COROLlaRY. If $k=3$, then $\left|k_{1}-k_{2}\right|=1$, and $\Gamma=G\left(k_{1}, k_{2}\right)$.

Proof. Since $\Gamma$ is 3-regular and connected, and $G\left(k_{1}, k_{2}\right)$ is a subgraph of $\Gamma$, we must have $\Gamma=G\left(k_{1}, k_{2}\right)$.

This result and the following one are also given in [3] where only $k=3$ is considered.

3.6 COROLlaRY. If $k=3$, then $G$ is reconstructible.

Proof. By Theorem 3.4 and Corollary 3.5, $\Gamma$ is completely determined if $k=3$; it is either a prism or a polygon with main diagonals. This determines $G$ and $H$ uniquely. But then $G$ and $H$ are found not to be reconstructions of each other [3], a contradiction. We conclude that $G$ is reconstructible.

The case $k=4$ is somewhat more difficult. If $\left|k_{1}-k_{2}\right|=1$, then $\Gamma \neq$ $G\left(k_{1}, k_{2}\right)$, but only contains $G\left(k_{1}, k_{2}\right)$ as a proper subgraph. There is little that can be said without more information. Similarly, if $k \geqslant 5$, then all we know is that $G\left(k_{1}, k_{2}\right)$ is a subgraph of $\Gamma$. 
But if $k=4$ and $\left|k_{1}-k_{2}\right| \geqslant 2$, then by Theorem 3.4, $\Gamma=G\left(k_{1}, k_{2}\right)$ and the structure of $\Gamma$ is completely determined. Thus the graphs $G$ and $H$ are completely determined, and if there is a non-reconstructible graph of this type, it must be one of the $G$ 's or $H$ 's in this family.

It is not an easy matter to show that $G$ and $H$ are reconstructible in this case, and this illustrates an interesting point. Given two very similar graphs, how does one decide if they are reconstructions of each other or not? As Section 4 indicates, this can be a difficult question to answer.

\section{Reconstructing $G$ and $H$}

In this section we assume that $k=4,\left|k_{1}-k_{2}\right| \geqslant 2$, and that $\Gamma=G\left(k_{1}, k_{2}\right)$, and we show that $G$ and $H$ are in fact reconstructible graphs. We begin with several remarks about the structure of $G\left(k_{1}, k_{2}\right)$. As above, $g_{1}=\operatorname{gcd}\left(k_{1}+k_{2}+1\right)$ and $g_{2}=\operatorname{gcd}\left(k_{1}+1, k_{2}\right)$.

\subsection{LEMMA. $\operatorname{gcd}\left(g_{1}, g_{2}\right)=1$.}

Proof. If $g=\operatorname{gcd}\left(g_{1}, g_{2}\right)$, then $g \mid k_{1}$ and $g \mid k_{1}+1$, so $g=1$.

If we write $x g_{1}+y g_{2}=1$, where $x$ and $y$ are integers, then we can write

$$
\begin{aligned}
k_{1} & =a g_{1} g_{2}-x g_{1}, \\
k_{2} & =b g_{1} g_{2}-y g_{2}, \\
k_{1}+k_{2}+1 & =(a+b) g_{1} g_{2},
\end{aligned}
$$

where $a$ and $b$ are appropriate integers. These are useful expressions for finding a $k_{1}$ and $k_{2}$, once $g_{1}$ and $g_{2}$ are given, since the structure of $G\left(k_{1}, k_{2}\right)$ is better understood in terms of $g_{1}$ and $g_{2}$ than in terms of $k_{1}$ and $k_{2}$.

$G\left(k_{1}, k_{2}\right)$ consists of $g_{1}$ cycles of length $(a+b) g_{2}$ and $g_{2}$ cycles of length $(a+b) g_{1}$. The two sets of cycles intersect each other regularly, almost like a cartesian product. In fact, if $a+b=1$, so that $k_{1}+k_{2}+1=g_{1} g_{2}$, it is easy to see that $G\left(k_{1}, k_{2}\right)$ is isomorphic to the cartesian product $C g_{1} \times C g_{2}$ of two cycles.

We call the first set of cycles the $g_{1}$-cycles, and the second set the $g_{2}$-cycles. Edges of the $g_{1}$-cycles are called $g_{1}$-edges of $G\left(k_{1}, k_{2}\right)$, and edges of the $g_{2}$-cycles are called $g_{2}$-edges. Thus the edges of $G\left(k_{1}, k_{2}\right)$ are partitioned into $g_{1}$-edges and $g_{2}$-edges.

$G\left(k_{1}, k_{2}\right)$ is always embeddable on the torus, in which form its structure is graphically visible. This is illustrated in Figure 4.1. 


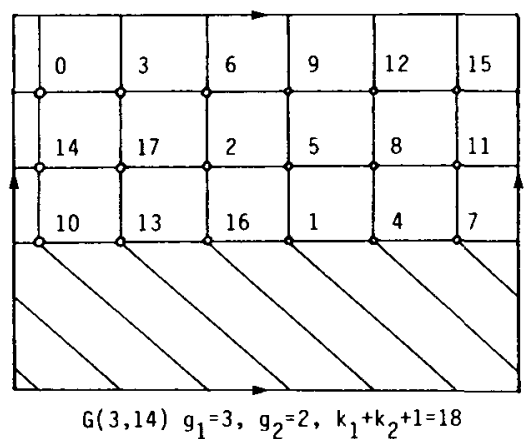

Figure 4.1

We call this the $g_{1}$-drawing of $G\left(k_{1}, k_{2}\right)$. The $g_{1}$-cycles appear as straight horizontal lines, and the $g_{2}$-cycles appear as vertical lines which must "wrap around" the torus several times in order to complete the cycle. In the $g_{2}$-drawing, these properties are reversed.

We define the "wrap around", $w_{1}$ and $w_{2}$, as follows. Let $N=k_{1}+k_{2}+1$.

4.2 Definition. In the $g_{1}$-drawing, the wrap around is $w_{1}$, where

$$
g_{1} k_{2} \equiv w_{1} k_{1}(\bmod N),
$$

and

$$
-N / 2 g_{1}<w_{1} \leqslant N / 2 g_{1} .
$$

In the $g_{2}$-drawing, the wrap around is $w_{2}$, where

$$
g_{2} k_{1} \equiv w_{2} k_{2}(\bmod N),
$$

and

$$
-N / 2 g_{2}<w_{2}<N / 2 g_{2}
$$

In words, $w_{1}$ is the amount by which the $g_{2}$-cycles wrap around in the $g_{1}$-drawing each time they go around the torus. For example, referring to Figure 4.1, we see that $w_{1}=2$.

It is easy to see that the formulas of Definition 4.2 define the appropriate quantity. If we examine the congruence

$$
\mathrm{g}_{1} k_{2} \equiv w_{1} k_{1} \quad(\bmod N),
$$

we see that $g_{1} \mid k_{1}$, and $g_{1} \mid N$, so that

$$
k_{2} \equiv w_{1} \frac{k_{1}}{g_{1}} \quad\left(\bmod N / g_{1}\right),
$$

so that we can, in fact, take $-N / 2 g_{1}<w_{1} \leqslant N / 2 g_{1}$. Now $g_{2} \mid k_{2}$ and $g_{2} \mid N / g_{1}$ by Lemma 4.1, and $\operatorname{gcd}\left(g_{2}, k_{1} / g_{1}\right)=1$. It follows that $g_{2} \mid w_{1}$, and we can write 
$w_{1}=a_{1} g_{2}$, which gives:

4.3 LEMMA. $w_{1}$ and $w_{2}$ satisfy:

(i) $w_{1}=a_{1} g_{2}$ and $w_{2}=a_{2} g_{1}$, for integers $a_{1}$ and $a_{2}$,

(ii) $k_{2} / g_{2} \equiv a_{1} k_{1} / g_{1}\left(\bmod N / g_{1} g_{2}\right)$,

(iii) $k_{1} / g_{1} \equiv a_{2} k_{2} / g_{2}\left(\bmod N / g_{1} g_{2}\right)$,

(iv) $-N / 2 g_{1} g_{2}<a_{i} \leqslant N / 2 g_{1} g_{2}$, for $i=1,2$.

It is easy to see that these congruences uniquely determine $a_{1}$ and $a_{2}$, and hence $w_{1}$ and $w_{2}$, since $\operatorname{gcd}\left(k_{1} / g_{1}, N / g_{1} g_{2}\right)=\operatorname{gcd}\left(k_{2} / g_{2}, N / g_{1} g_{2}\right)=1$. We make several further observations.

4.4 LeMma. $G\left(k_{1}, k_{2}\right)$ is a cartesian product if and only if $w_{1}=0$ or $w_{2}=0$.

4.5 Lemma. If $g_{i}=1$, then $\left|w_{i}\right| \neq 1$, for $i=1,2$.

PROoF. If $g_{i}=1$ and $\left|w_{i}\right|=1$, this would imply multiple edges in $G\left(k_{1}, k_{2}\right)$, or that $k_{1}=k_{2}$, a contradiction.

\subsection{LEMMA. If $g_{1}=g_{2}=1$, then $\left|w_{1}\right| \neq\left|w_{2}\right|$.}

Proof. If $\left|w_{1}\right|=\left|w_{2}\right|$, then this would imply that the $g_{1}$-drawing and $g_{2}$-drawing of $G\left(k_{1}, k_{2}\right)$ were identical, which would imply that $G \cong H$. But since we assume that $G \supsetneqq H$, this is a contradiction.

Finally, we mention that as well as the automorphism $\theta: u_{i} \rightarrow u_{i+1}, G\left(k_{1}, k_{2}\right)$ admits a dihedral flip $\rho: u_{i} \rightarrow u_{-i}$, where the subscript is computed modulo $k_{1}+k_{2}+1$. The existence of $\rho$ is most easily seen from Definition 3.2. It preserves the edge-orbits of $\langle\theta\rangle$.

In order to prove that $G$ and $H$ are reconstructible, we use the following approach. If $X$ is a graph with fewer vertices than $G$, let $s(G, X)$ denote the number of induced subgraphs of $G$ which are isomorphic to $X$, that is,

$$
s(G, X)=\left|\left\{V^{\prime} \subseteq V(G) \mid G\left[V^{\prime}\right] \cong X\right\}\right| .
$$

To say that $G$ and $H$ are reconstructions of each other is to say that $s(G, X)=s(H, X)$, for every $X$ such that $\nu(X)<\nu(G)$ (see [2]). In order to prove that $G$ and $H$ are not reconstructions of each other, we find some $X$ such that $s(G, X) \neq s(H, X)$. Since $H$ is the only possible reconstruction of $G$, it will follow that $G$ and $H$ are in fact reconstructible graphs. 
We know that $\Gamma=G\left(k_{1}, k_{2}\right)$, and that $G=\Gamma-u_{0} u_{k_{1}}$ and $H=\Gamma-u_{0} u_{k_{2}}$, where we take $V(G)=V(H)=\left\{u_{0}, u_{1}, \ldots, u_{k_{1}+k_{2}+1}\right\}$. If $V^{\prime} \subseteq V(G)$ such that $G\left[V^{\prime}\right] \cong X$, then as long as $V^{\prime}$ does not contain any of $u_{0}, u_{k_{1}}$, or $u_{k_{2}}$, we will also have $H\left[V_{k_{1}}^{\prime}\right] \cong X$. The same will be true if $V^{\prime}$ contains only one of $u_{0}, u_{k_{1}}, u_{k_{2}}$, or if $V^{\prime}$ contains $u_{k_{1}}$ and $u_{k_{2}}$, but not $u_{0}$. We can accordingly restrict the set $V^{\prime}$ to subsets of $V(G)$ which contain $u_{0}$, and at least one of $u_{k_{1}}$ and $u_{k_{2}}$. We make the following definition.

4.7 Definition. Given a graph $X$, we define

$$
s^{\prime}(G, X)=\mid\left\{V^{\prime} \subseteq V(G) \mid u_{0} \in V^{\prime},\left\{u_{k_{1}}, u_{k_{2}}\right\} \cap V^{\prime} \neq \varnothing \text {, and } G\left[V^{\prime}\right] \cong X\right\} \mid \text {. }
$$

A similar definition holds for $s^{\prime}(H, X)$.

In order to show that $G$ and $H$ are not reconstructions of each other, we will find a graph $X$ such that $s^{\prime}(G, X) \neq s^{\prime}(H, X)$. This is most easily done in terms of the parameters $g_{1}, g_{2}, w_{1}$, and $w_{2}$. There are many special cases which must be examined, and so the proof of the desired result follows through a sequence of lemmas.

4.8 Lemma. If $\left|w_{1}\right|=1$ and $g_{1}=2$, then $G$ and $H$ are not reconstructions of each other.

Proof. Let $X$ be the graph in Figure 4.2.

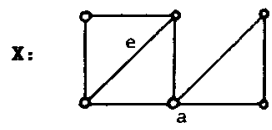

Figure 4.2

We consider two cases: $w_{1}=1$ and $w_{1}=-1$.

Case 1. $w_{1}=1$. The graph $\Gamma=G\left(k_{1}, k_{2}\right)$ is of the form indicated in Figure 4.3. Since all graphs on 9 or fewer vertices are known to be reconstructible (see [1]) we can assume that $N / g_{1} \geqslant 5$.

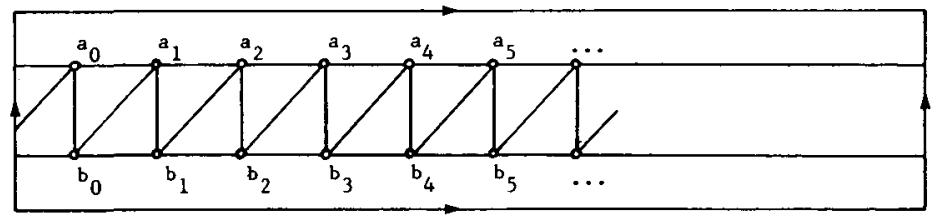

Figure 4.3 
Notice that any $g_{1}$-edge of $\Gamma$ has exactly one path of length 2 joining its endpoints, but that a $g_{2}$-edge has exactly 2 paths of length 2 joining its endpoints. Since the edge $e$ of $X$ has two such 2-paths, any embedding of $X$ into $G$ or $H$ must have $e$ as a $g_{2}$-edge. Without loss of generality, we can take this edge to be $a_{3} b_{2}$. Thus the vertex $a$ of $X$ must correspond either to $a_{2}$ or to $b_{3}$. Clearly, this gives no embeddings of $X$ into $\Gamma$. But if the edges $b_{1} b_{2}$ or $a_{3} a_{4}$ were deleted, there would be an embedding. But $\Gamma-b_{1} b_{2} \cong \Gamma-a_{3} a_{4} \cong G$, so that $s^{\prime}(G, X)=2$, whereas $s^{\prime}(H, X)=0$. This is because all $g_{1}$-edges are similar, all $g_{2}$-edges are similar, and deleting an edge of $\Gamma$ cannot increase the number of 2-paths. It follows that $G$ and $H$ are not reconstructions of each other.

Case 2. $w_{1}=-1$. It is easy to verify that in this case the graph $\Gamma$ is isomorphic to that when $w_{1}=1$. The same argument applies.

4.9 LEMMA. If $\left|w_{1}\right|=2$ and $g_{1}=1$, then $G$ and $H$ are not reconstructions of each other.

Proof. Let $X$ denote the graph in Figure 4.4.

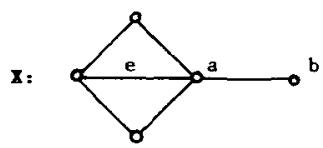

Figure 4.4

Case 1. $w_{1}=2$. The graph $\Gamma$ is illustrated in Figure 4.5.

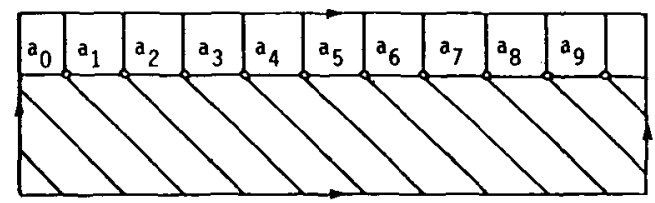

Figure 4.5

Since all graphs on 9 or fewer vertices are reconstructible, we can take $N / g_{1} \geqslant 10$.

Notice that any $g_{1}$-edge $a_{2} a_{3}$ of $\Gamma$ has exactly two 2-paths joining its endpoints: $a_{2} a_{1} a_{3}$ and $a_{2} a_{4} a_{3}$. But a $g_{2}$-edge $a_{2} a_{4}$ has exactly one such 2-path: $a_{2} a_{3} a_{4}$. It follows that any embedding of $X$ into $\Gamma$ must have $e$ as a $g_{1}$-edge, say $a_{2} a_{3}$.

The vertex $a$ of $X$ must then correspond to $a_{2}$ or $a_{3}$, and $b$ must correspond to $a_{1}$ or $a_{5}$, respectively. But $a_{0} a_{1} \in E(\Gamma)$ and $a_{3} a_{5} \in E(\Gamma)$. It follows that $X$ is a subgraph of $\Gamma-a_{0} a_{1}$ and of $\Gamma-a_{3} a_{5}$. But $\Gamma-a_{0} a_{1} \cong \Gamma-a_{3} a_{5} \cong G$, so that $s^{\prime}(G, X)=2$ and $s^{\prime}(H, X)=0$. Thus $G$ and $H$ are not reconstructions of each other. 
Case 2. $w_{1}=-2$. The arguments are almost identical to Case 1. We again have $s^{\prime}(G, X)=2$ and $s^{\prime}(H, X)=0$.

4.10 LEMMA. If $\left|w_{1}\right|=1$ and $g_{1}=3$, then $G$ and $H$ are not reconstructions of each other.

Proof. Let $X$ denote the graph in Figure 4.6.

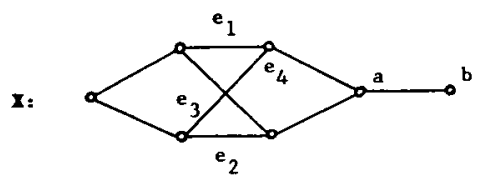

Figure 4.6

As before, we have two cases.

Case 1. $w_{1}=1$. The graph $\Gamma$ is illustrated below in Figure 4.7.

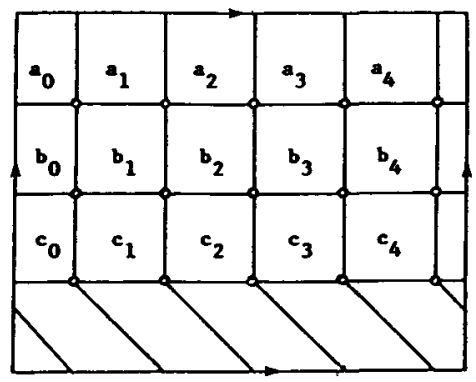

Figure 4.7

Since all graphs on 9 or fewer vertices are reconstructible, we can take $N / g_{1} \geqslant 4$. Consider a $g_{2}$-edge $a_{2} b_{2}$. The number of 3-paths joining $a_{2}$ to $b_{2}$ is five: $a_{2} a_{1} b_{1} b_{2}, a_{2} c_{1} b_{1} b_{2}, a_{2} c_{1} c_{2} b_{2}, a_{3} c_{3} c_{2} b_{2}$, and $a_{2} a_{3} b_{3} b_{2}$. For a $g_{1}$-edge $a_{2} a_{3}$, the number of 3-paths is 3 if $N / g_{1} \geqslant 5: a_{2} b_{2} b_{3} a_{3}, a_{2} b_{2} c_{2} a_{3}$, and $a_{2} c_{1} c_{2} a_{3}$. If $N / g_{1}=4$, there is a fourth path: $a_{2} a_{1} a_{0} a_{3}$.

In the graph $X, e_{1}, e_{2}, e_{3}$, and $e_{4}$ are all similar edges. Their endpoints each have three 3-paths connecting them, two of which are internally disjoint, with the third intersecting them both. In any embedding of $X$ into $\Gamma$, not all of $e_{1}, e_{2}, e_{3}$, and $e_{4}$ can appear as $g_{2}$-edges; for no four $g_{2}$-edges form a cycle. Therefore, we can take $e_{1}$ as a $g_{1}$-edge in some embedding, and we can take this edge to be $a_{2} a_{3}$.

If we examine the three (possibly four) 3-paths joining $a_{2}$ to $a_{3}$, we see there is only one way of choosing them so as to conform to the structure of $X$ mentioned above. This is illustrated in Figure 4.8. 


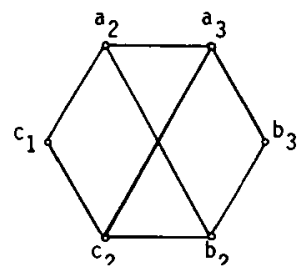

Figure 4.8

The vertex $a$ of $X$ must correspond either to $c_{1}$ or to $b_{3}$. If $a$ corresponds to $c_{1}$, then $b$ must correspond to $b_{1}$ or $c_{0}$. If $a$ corresponds to $b_{3}$, then $b$ must correspond to $c_{3}$ or $b_{4}$. We are interested in embeddings of $X$ into $G$ or $H$ such that $V(X)$ contains $u_{0} \in V(\Gamma)$ and at least one of $\left\{u_{k_{1}}, u_{k_{2}}\right\}$. If we consider the four possible combinations for $a$ and $b$, we find that $s^{\prime}(G, X)=6$ and $s^{\prime}(H, X)$ $=2$, so that $G$ and $H$ are not reconstructions of each other.

Case 2. $w_{1}=-1$. The analysis is very similar to Case 1 . We obtain $s^{\prime}(G, X)=7$ and $s^{\prime}(H, X)=3$.

4.11 LEMMA. If $\left|w_{1}\right|=2$ and $g_{1}=2$, then $G$ and $H$ are not reconstructions of each other.

Proof. Let $X$ denote the graph in Figure 4.9.

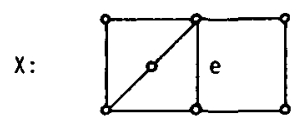

Figure 4.9

Case 1. $w_{1}=2$. The graph $\Gamma$ is illustrated in Figure 4.10. As before we can assume that $N / g_{1} \geqslant 5$.

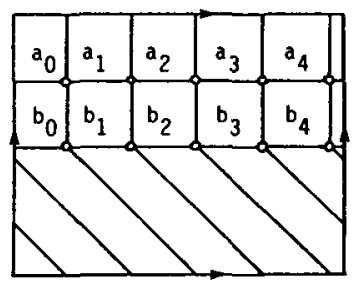

Figure 4.10

Notice that a $g_{2}$-edge $a_{2} b_{2}$ has four 3-paths connecting its endpoints $a_{2} a_{1} b_{1} b_{2}$, $a_{2} b_{0} b_{1} b_{2}, a_{2} a_{3} b_{1} b_{2}$, and $a_{2} a_{3} b_{3} b_{2}$. A $g_{1}$-edge $a_{2} a_{3}$ has five such 3-paths: $a_{2} b_{2} b_{3} a_{3}$, 
$a_{2} b_{2} a_{4} a_{3}, a_{2} b_{2} b_{1} a_{3}, a_{2} a_{1} b_{1} a_{3}$, and $a_{2} b_{0} b_{1} a_{3}$. Consider the edge $e$ of $X$. Its endpoints are connected by three 3-paths, two of which intersect and which are disjoint from the third. Suppose there is an embedding of $X$ into $\Gamma$ in which $e$ appears as a $g_{2}$-edge, say $e=a_{2} b_{2}$. Examining the 3-paths joining $a_{2}$ to $b_{2}$ listed above, we see that we must take $X$ as in Figure 4.11 ; there are no other possibilities.

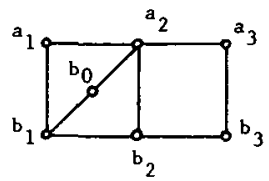

Figure 4.11

But in $\Gamma, b_{1} a_{3} \in E(\Gamma)$. Therefore $X$ is a subgraph of $\Gamma-b_{1} a_{3} \cong H$.

Now suppose there is an embedding of $X$ in which $e$ appears as a $g_{1}$-edge $a_{2} a_{3}$. If we examine the 3-paths listed above, we see there are only four possible embeddings (Figure 4.12).
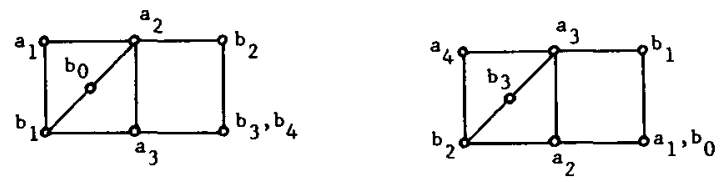

Figure 4.12

The doubly labelled vertices can be labelled either way. But $b_{1} b_{2} \in E(\Gamma)$. Thus $X$ is a subgraph of $\Gamma-b_{1} b_{2} \cong G$. It follows that $s^{\prime}(G, X)=4$, and $s^{\prime}(H, X)=1$, so that $G$ and $H$ are not reconstructions of each other.

Case 2. $w_{1}=-2$. The arguments are almost identical to Case 1. We obtain $s^{\prime}(G, X)=4$ and $s^{\prime}(H, X)=1$.

4.12 LEMMA. If $g_{1}=g_{2}=1$, and $\left|w_{2}\right|>\left|w_{1}\right|=3$, then $G$ and $H$ are not reconstructions of each other.

Proof. Let $X$ denote the graph in Figure 4.13.

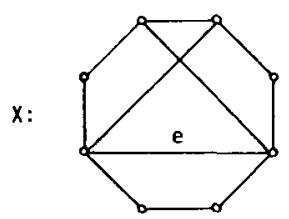

Figure 4.13 
Case 1. $w_{1}=3$. The graph $\Gamma$ is illustrated in Figure 4.14.

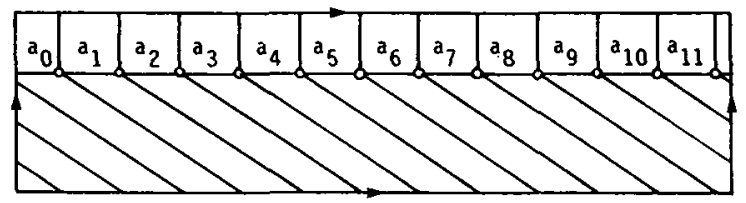

Figure 4.14

We can assume that $N / g_{1} \geqslant 10$. Consider a $g_{1}$-edge $a_{3} a_{4}$ of $\Gamma$. There are five 3-paths joining $a_{3}$ to $a_{4}: a_{3} a_{2} a_{1} a_{4}, a_{3} a_{2} a_{5} a_{4}, a_{3} a_{0} a_{5} a_{4}, a_{3} a_{6} a_{7} a_{4}$, and $a_{3} a_{0} a_{1} a_{4}$. To show that these are all the 3-paths joining $a_{3}$ to $a_{4}$, notice that $g_{2}=1$. Thus $a_{3}$ and $a_{4}$ are in the same $g_{2}$-cycle of $\Gamma$. If there were a 3-path from $a_{3}$ to $a_{4}$ using only $g_{2}$-edges, it would follow that $\left|w_{2}\right|=3$. But $\left|w_{2}\right| \geqslant 4$, so that every 3-path from $a_{3}$ to $a_{4}$ must use a $g_{1}$-edge. Since $N / g_{1} \geqslant 10$, we obtain the desired result by inspecting Figure 4.14.

A $g_{2}$-edge $a_{3} a_{6}$ has three 3-paths joining its endpoints: $a_{3} a_{4} a_{5} a_{6}, a_{3} a_{4} a_{7} a_{6}$, and $a_{3} a_{2} a_{5} a_{6}$.

Consider the edge $e$ of $X$. In $X$, there are three mutually internally disjoint 3-paths joining its endpoints. So in any embedding of $X$ into $\Gamma, e$ must appear as a $g_{1}$-edge, $a_{3} a_{4}$ say. By examining the five 3-paths listed above, we see there is only one such set of 3-paths, giving two ways of embedding $X$, as shown in Figure 4.15.
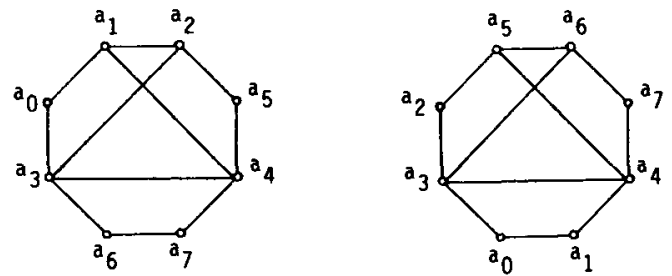

Figure 4.15

But in the first case $a_{5} a_{6} \in E(\Gamma)$ and in the second case $a_{1} a_{2} \in E(\Gamma)$. If $N>10$, there are no other edges induced by these vertices. We conclude that if $N>10$, then $X$ is a subgraph of $\Gamma-a_{5} a_{6}$ and of $\Gamma-a_{1} a_{2}$. But $\Gamma-a_{5} a_{6} \cong \Gamma-$ $a_{1} a_{2} \cong G$, so that $s^{\prime}(G, X)=2$, and $s^{\prime}(H, X)=0$.

If $N=10$ and $w_{1}=3$, it is easy to check that we must also have $\left|w_{2}\right|=3$, a contradiction. It follows that $G$ and $H$ are not reconstructions of each other.

Case 2. $w_{1}=-3$. The analysis is identical. We again have $s^{\prime}(G, X)=2$ and $s^{\prime}(H, X)=0$. 
4.13 LEMMA. If $g_{1}=g_{2}=1$ and $\left|w_{1}\right|>\left|w_{2}\right| \geqslant 4$, then $G$ and $H$ are not reconstructions of each other.

Proof. We define a graph $Y(m)$, for any integer $m \geqslant 2$, consisting of two paths of length $m-1$, with corresponding vertices joined by a matching. $Y(5)$ is illustrated in Figure 4.16.

$Y(5)$

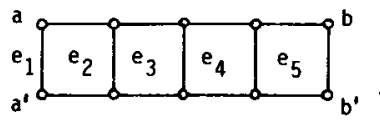

Figure 4.16

Let $X=Y\left(\left|w_{1}\right|\right)$. Notice that in $\Gamma$, any $g_{1}$-edge or $g_{2}$-edge has exactly two 3-paths joining its endpoints. This is because $\left|w_{1}\right|,\left|w_{2}\right| \geqslant 4$. The edge $e_{2}$ of $X$ also has this property. This if $e_{2}$ is embedded into $\Gamma$ as a $g_{1}$-edge, or $g_{2}$-edge, the embedding of $e_{1}$ and $e_{3}$ is essentially forced, and this in turn forces the rest of the embedding. But since $X=Y\left(\left|w_{1}\right|\right)$ and $\left|w_{1}\right|>\left|w_{2}\right|$, it is easy to see there are no embeddings of $X$ into $G$ or $H$ if $e_{2}$ is chosen as a $g_{1}$-edge. There are too many additional edges induced by these vertices.

But if $e_{2}$ is chosen as a $g_{2}$-edge, the resulting embedding has only one extra edge, either $a b^{\prime}$ or $a^{\prime} b$, if $\left|w_{1}\right|<\left[N / 2 g_{1}\right]$; and two extra edges, $a b^{\prime}$ and $a^{\prime} b$, if $\left|w_{1}\right|=\left[N / 2 g_{1}\right]$. In the first case, $X$ is a subgraph of $\Gamma-a b^{\prime} \cong G$, so that $s^{\prime}(G, X)=1$ and $s^{\prime}(H, X)=0$. In the second case, $X+a^{\prime} b$ is a subgraph of $\Gamma-a b^{\prime} \cong G$, but not of $H$, so that $s^{\prime}\left(G, X+a^{\prime} b\right)=1$ and $s^{\prime}\left(H, X+a^{\prime} b\right)=0$. In either case, $G$ and $H$ are not reconstructions of each other.

4.14 LeMMA. If $g_{1}=1, g_{2}=2,\left|w_{1}\right| \geqslant 4$, and $\left|w_{2}\right| \geqslant 3$, then $G$ and $H$ are not reconstructions of each other.

Proof. With these values of the parameters, it is quite easy to see that any edge of $\Gamma$ has exactly two 3-paths joining its endpoints, since $N \geqslant 10$. We look at three cases.

Case 1. $\left|w_{2}\right| \geqslant\left|w_{1}\right|$. Let $X=Y\left(\left|w_{2}\right|+1\right)$. It is easy to see that there are no embeddings of $X$ into $G$ or $H$ if the edge $e_{2}$ of $X$ appears as a $g_{2}$-edge since $\left|w_{2}\right|+1>\left|w_{1}\right|$. But if $e_{2}$ appears as a $g_{1}$-edge, then $X$ embeds into $\Gamma$ with a single additional induced edge, $a b^{\prime}$ or $a^{\prime} b$. So $X$ is a subgraph of $\Gamma-a^{\prime} b$ or of $\Gamma-a b^{\prime}$, both of which are isomorphic to $G$. Thus, $s^{\prime}(G, X)=1$ and $s^{\prime}(H, X)=0$.

Case 2. $\left|w_{1}\right|=\left|w_{2}\right|+1$. Let $X=Y\left(\left|w_{1}\right|\right)=Y\left(\left|w_{2}\right|+1\right)$. As in Case 1, there is an embedding of $X$ into $G$ when $e_{2}$ is a $g_{1}$-edge, so that $s^{\prime}(G, X) \geqslant 1$. If $X$ is embedded into $\Gamma$ with $e_{2}$ appearing as a $g_{2}$-edge, then only a single extra edge is 
induced, $a b^{\prime}$ or $a^{\prime} b$, both of which are $g_{1}$-edges. It follows that $X$ is a subgraph of $G$ in this case. So $s^{\prime}(G, X)=2$ and $s^{\prime}(H, X)=0$.

Case 3. $\left|w_{1}\right|>\left|w_{2}\right|+1$. Let $X=Y\left(\left|w_{1}\right|\right)$. There are no embeddings of $X$ into $G$ or $H$ if $e_{2}$ appears as a $g_{1}$-edge. If $e_{2}$ appears as a $g_{2}$-edge, there will be an embedding, with one extra edge induced, a $g_{1}$-edge. Thus $X$ is a subgraph of $G$, so that $s^{\prime}(G, X)=1$ and $s^{\prime}(H, X)=0$.

4.15 LEMMA. If $g_{1} \geqslant 3$ and $\left|w_{1}\right| \geqslant 2$, then $G$ and $H$ are not reconstructions of each other.

Proof. We define a graph $W(m)$ for any integer $m \geqslant 3$, consisting of two cycles of length $m$, with corresponding vertices joined by a matching. $W(5)$ is illustrated in Figure 4.17.

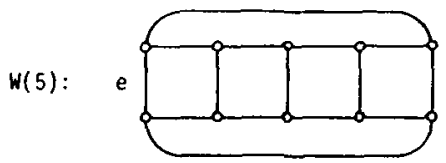

Figure 4.17

Notice that the edge $e$ of $W(m)$ has exactly two 3-paths connecting its endpoints. Let $X=W\left(N / g_{1}\right)$. This is possible, since $N / g_{1} \geqslant 3$; for otherwise the $g_{1}$-cycles of $\Gamma$ would have length at most two.

It is easy to see that any $g_{2}$-edge of $\Gamma$ has exactly two 3-paths joining its endpoints. If $N / g_{1} \neq 4$, then any $g_{1}$-edge of $\Gamma$ also has this property. Thus if $N / g_{1} \neq 4$, any embedding of $X$ into $\Gamma$ is forced, once the edge $e$ has been embedded. It is easy to see that $X$ can be embedded in $\Gamma$ with $e$ as a $g_{2}$-edge, but not as a $g_{1}$-edge. This gives $s^{\prime}(G, X)=0$ and $s^{\prime}(H, X)=N / g_{1}$, if $N / g_{1} \neq 4$. If $N / g_{1}=4$, then a $g_{1}$-edge of $\Gamma$ has exactly three mutually internally disjoint 3-paths connecting its endpoints. But in this case $X=W(4)$ is the graph of the cube, which is transitive on edges, so that we can assume, without loss of generality, that any embedding of $X$ into $\Gamma$ has $e$ as a $g_{2}$-edge. The embedding is then forced, as above, which gives $s^{\prime}(G, X)=0$ and $s^{\prime}(H, X)=N / g_{1}$. Thus $G$ and $H$ are not reconstructions of each other.

4.16 LEMMA. If $w_{1}=w_{2}=0$, then $G$ and $H$ are not reconstructions of each other.

Proof. By Lemma 4.1, $\operatorname{gcd}\left(g_{1}, g_{2}\right)=1$, so $g_{1} \neq g_{2}$. Without loss of generality, take $g_{1}>g_{2} \geqslant 3$, and let $X=W\left(N / g_{1}\right)$. We use the same arguments as Lemma 4.15 to show that $G$ and $H$ are not reconstructions of each other. 
4.17 THEOREM. If $k=4$ and $\left|k_{1}-k_{2}\right| \geqslant 2$ then $G$ is reconstrucible.

Proof. We know that if $G$ is not reconstructible, then $G$ and $H$ are reconstructions of each other, where $G=\Gamma-u_{0} u_{k_{1}}, H=\Gamma-u_{0} u_{k_{2}}$, and $\Gamma=G\left(k_{1}, k_{2}\right)$.

If $\Gamma$ is a cartesian product $C_{g_{1}} \times C_{g_{2}}$, then $w_{1}=w_{2}=0$. By Lemma 4.16, $G$ and $H$ are not reconstructions of each other. Otherwise, by Lemma $4.4, w_{1} \neq 0 \neq w_{2}$. Thus $g_{1}+\left|w_{1}\right| \geqslant 2$.

Case 1. $g_{1}+\left|w_{1}\right|=2$. By Lemma 4.5, this is impossible.

Case 2. $g_{1}+\left|w_{1}\right|=3$. There are two possibilities: $g_{1}=2$ and $\left|w_{1}\right|=1$ or $g_{1}=1$ and $\left|w_{1}\right|=2$. By Lemmas 4.8 and $4.9, G$ and $H$ are not reconstructions of each other.

Case 3. $g_{1}+\left|w_{1}\right|=4$. There are three possibilities: $g_{1}=3$ and $\left|w_{1}\right|=1, g_{1}=$ $\left|w_{1}\right|=2$, or $g_{1}=1$ and $\left|w_{1}\right|=3$. The first two possiblities are dealt with by Lemmas 4.10 and 4.11 .

For the third possibility, notice that by Lemma $4.3, g_{2} \mid w_{1}$. Therefore, $g_{2}=1$ or $g_{2}=3$. Suppose first that $g_{2}=1$. If $\left|w_{2}\right|=2$, then we can reverse the roles of $g_{1}$ and $g_{2}$ and use Lemma 4.8. It's impossible to have $\left|w_{2}\right|=3$, by Lemma 4.6. If $\left|w_{2}\right| \geqslant 4$, then we use Lemma 4.12 .

Suppose next that $g_{2}=3$. If $\left|w_{2}\right|=1$, then we reverse the roles of $g_{1}$ and $g_{2}$, and use Lemma 4.10. If $\left|w_{2}\right| \geqslant 2$, then we interchange $g_{1}$ and $g_{2}, w_{1}$ and $w_{2}$, and use Lemma 4.15. Thus, in all cases, $G$ and $H$ are not reconstructions of each other.

Case 4. $g_{1}+\left|w_{1}\right| \geqslant 5$, Consider $g_{2}+\left|w_{2}\right|$. If $g_{2}+\left|w_{2}\right| \leqslant 4$, then we can reverse the roles of $g_{1}$ and $g_{2}$, and use one of Cases 1,2 , or 3 above. So we can assume that $g_{2}+\left|w_{2}\right| \geqslant 5$. If $g_{1}=g_{2}=1$, then by Lemma 4.13 we are done. If $g_{1}=1$ and $g_{2}=2$, then we use Lemma 4.14. Otherwise both of $g_{1}$ and $g_{2}$ are at least 2, so one of them, say $g_{1}$, is at least 3. We then use Lemma 4.15.

We see that in all cases, $G$ and $H$ are not reconstructions of each other. It follows that $G$ is reconstructible.

\section{Concluding remarks}

The fact that $\theta$ is a partial automorphism of $\Gamma$ forces much of the structure of $G$ and $H$. In some cases it determines $G$ and $H$ completely. In the cases for which only some of the structure of $G$ and $H$ has been determined, it would seem natural to set $G-a \cong H-b$ for appropriate vertices $a$ and $b$ of degree $k$, in order to get a second mapping $\theta^{\prime}$. If we can find an appropriate graph $\Gamma^{\prime}$ such that $\theta$ and $\theta^{\prime}$ extend to automorphisms of $\Gamma^{\prime}$, then the group $\left\langle\theta, \theta^{\prime}\right\rangle$ must act on $\Gamma^{\prime}$. This would force much more of the structure of $G$ and $H$, and may make it possible to produce a counterexample to the reconstruction conjecture. 


\section{References}

[1] J. A. Bondy and R. L. Hemminger, 'Graph reconstruction-a survey', J. Graph Theory 1 (1977), 227-268.

[2] W. L. Kocay, 'Some new methods in reconstruction theory', Proceedings of the Ninth Australian Combinatorial Conference, (Springer-Verlag), to appear.

[3] W. L. Kocay, 'Partial automorphisms and the reconstruction of bi-degreed graphs', Congr. Numer, to appear.

\section{Department of Mathematics}

The University of Manitoba

Winnipeg, Manitoba

Canada 